\title{
Pediatric cancer spectrum in Kenya: a histopathologic review
}

\author{
Franklin C. Margaron • Dan Poenaru • \\ Alan Northcutt
}

Accepted: 16 June 2010/Published online: 1 July 2010

(C) Springer-Verlag 2010

\begin{abstract}
Background Pediatric malignancies constitute a unique health care burden especially in resource-poor settings such as Africa, and the use of external epidemiologic data in managing these children may lead to erroneous approaches. Methods A retrospective histopathologic analysis of 6,771 pathology reports between March 1992 and August 2006 was undertaken in children under the age of 18. Data were derived from the Kijabe Hospital pathology database, which receives pathologic specimens from 70 different mission hospitals, clinics, and regional hospitals throughout Kenya. Results 1,217 of these specimens represented malignancies. The top ten pediatric malignancies were Burkitt's lymphoma (16.6\%), non-Hodgkin's lymphoma (8.5\%), Hodgkin's lymphoma (7.6\%), Kaposi's sarcoma (7.6\%), osteosarcoma (7.3\%), gonadal germ-cell tumors (5.8\%), rhabdomyosarcoma $(3.5 \%)$, nephroblastoma $(3.4 \%)$, epithelial carcinoma (2.8\%), and chondrosarcoma (1.8\%).

Conclusion Comparison with several previous African and western pediatric cancer databases is undertaken, revealing significant trends useful for the care of children with cancer in the region.
\end{abstract}

Keywords Cancer - Oncology - Africa - Lymphoma . Sarcoma · Carcinoma · Hodgkin's · Burkitt's · Wilms'

\section{Introduction}

In 1964 Drs. Denis Burkitt and MSR Hutt traveled across Uganda, Tanzania, and Kenya to teach histopathologic

F. C. Margaron · D. Poenaru $(\bowtie) \cdot$ A. Northcutt BethanyKids at Kijabe Hospital, Box 20, Kijabe 00220, Kenya e-mail: dpoenaru@gmail.com technique and gather data on cancers endemic to East Africa [1]. They recognized then that the spectrum of cancer across these areas differed greatly from those seen in the West, and even differed from one area to another. Nearly half a century later, the understanding and treatment of pediatric cancer have advanced greatly worldwide-yet the true incidence and outcomes of cancers in East Africa are still largely unknown. Many children in these areas have limited access to diagnosis and therapy, thus affecting negatively both the demographics and outcomes of oncologic disease in the region.

The total incidence of cancer in children under the age of 15 has been shown to be relatively constant from one geographical location to another. This typically ranges between 1 and 2.5 per 1,000 children [2]. However, the actual geographical distribution of the types of cancers represented varies greatly from one area to the next [2]. Childhood cancers represent a diffuse spectrum of disease in which certain types of tumors can present in multiple anatomic locations. Therefore, childhood cancers are subtyped primarily by histology [3, 4].

Survival rates for all children with pediatric solid tumors have risen in developed countries from 27\% in 1960 to $70 \%$ in 1990 [5]. Survival rates for the non-Hodgkin's lymphomas, including the form that bears Dr. Burkitt's name, have improved greatly in the pediatric population-increasing from $56 \%$ in $1975-1984$ to $72 \%$ in 1985-1994. At the current time the survival for Hodgkin's lymphoma is over 90\% [6]. These data derived from Western databases such as the National Cancer Data Base and the Surveillance, Epidemiology, and End-Results (SEER) database give information on age-specific incidence, geographic distribution, risk factors, trends over time, and survival characteristics. The databases have led to knowledge sharing that has facilitated the rapid progress in the fight against pediatric cancer. 
Several African countries have benefited from national pediatric cancer databases, including Malawi [7] and Tanzania [8]. Other countries have incidence data derived from a single large city or institutional studies such as Uganda [2], Nigeria [9], and Zambia [10, 11]. Kenya currently has no national pediatric cancer database, and information on cancer in the country can only be inferred from a few studies. The first is a prospective study over a 6-month period at Kenyatta National Hospital and seven other centers, examining the distribution of 157 pediatric cancers seen during that time [12]. The second is a histopathologic review from three centers in Western Kenya between 1979 and 1994 covering 600 solid pediatric cancers [13]. Several specific diagnoses have been better explored in Kenya, including Burkitt's lymphoma [14-16] and Hodgkin's disease $[17,18]$. Our study was intended to broaden the understanding of the spectrum of pediatric cancers in Kenya.

\section{Materials and methods}

The Kijabe Hospital Pathology database is a collection of reports from histopathologic specimens sent to the hospital for diagnosis from 70 different mission hospitals, clinics, and regional hospitals from all over Kenya. Many of these locations can be seen on the map in Fig. 1 as blue squares. $3.6 \%$ of the total samples came from locations outside of Kenya-these reports were excluded from the final

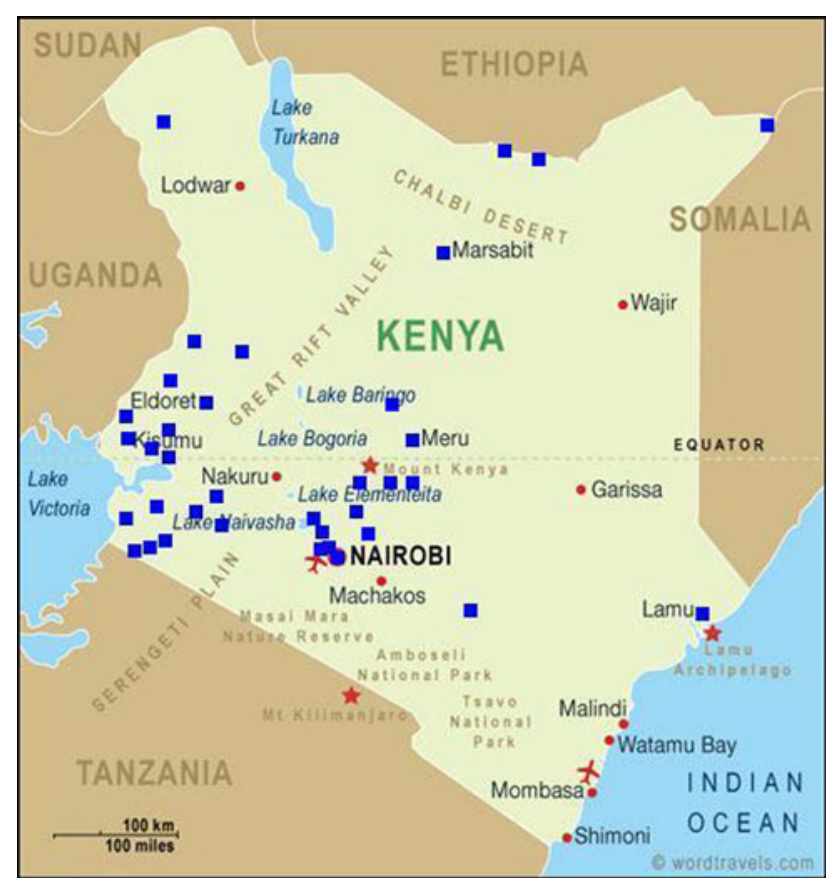

Fig. 1 Pathology referral centers in Kenya analysis. Data available on all reports included patient demographics, details of surgery performed, gross and microscopic description, and final diagnosis. Those patients with incomplete information or unreliable age identifiers were excluded.

All pathology samples were received in formalin saline solution at Kijabe Hospital. These were embedded in paraffin, sectioned, and stained with hematoxylin and eosin under standard technique. Other stains available included periodic acid-Schiff stain and AFB. Histopathologic analysis was performed by the pathology staff at Kijabe Hospital, which was mostly comprised of rotating short-term pathologists from the United States. Pathologic diagnosis was confirmed when necessary through telemedicine consultation with other members of the Kijabe pathology network overseas. When possible, samples requiring further staining and analysis were taken to centers where such testing could be performed.

\section{Results}

The total database from March 1992 to August 2006 comprised 65,536 reports. A total of 7,025 pediatric (under age 18) specimens were identified, of which 6,771 came from within Kenya. 1,217 of these samples were identified as malignancies.

The organization of pathology reports is shown in Table 1, using the 12 histopathologic categories established by the International Classification of Childhood Cancers (ICCC) specifications [4], with some expanded sub-typing of the soft-tissue sarcomas and carcinomas for clarity.

The top ten pediatric cancer diagnoses for several databases are presented in Table 2. When comparing the Kijabe database with the American SEER database [6], there are some notable differences. The top three diagnoses on the SEER database, acute lymphoid leukemia (24.5\%) astrocytoma (10\%), and neuroblastoma (7.5\%) are not even represented in the top ten cancers in the Kijabe database. Neither acute myeloid leukemia $(4.7 \%)$ nor peripheral neuroectodermal tumors $(4.5 \%)$ are represented, which are number 5 and 6, respectively, on the SEER database. In the Kijabe database the lymphomas, Burkitt's (16.6\%), nonHodgkin's (8.5\%), and Hodgkin's (7.6\%), make up the top three most frequent diagnoses. The SEER database, on the other side, has only one representative lymphoma, Hodgkin's disease $(4.4 \%)$, in the top ten. Other pediatric cancers which are more strongly represented in the Kijabe database as compared with SEER include Kaposi's sarcoma (7.5 vs. $0 \%$ ), osteosarcoma (7.4 vs. $2.4 \%)$, gonadal germ-cell tumors (5.8 vs. 1.7\%), and epithelial carcinoma (2.7 vs. $0 \%)$. 
Table 1 Kijabe Hospital pathology database for pediatric cancers

\begin{tabular}{|c|c|c|}
\hline & Cases & $\begin{array}{l}\text { Total } \\
(\%)\end{array}$ \\
\hline Total cancers & 1,217 & 100.0 \\
\hline Leukemia & 30 & 2.5 \\
\hline Acute lymphoid & 11 & 0.9 \\
\hline Acute myeloid & 10 & 0.8 \\
\hline Chronic lymphocytic & 3 & 0.2 \\
\hline Unspecified & 6 & 0.5 \\
\hline Lymphomas and reticuloendothelial neoplasms & 420 & 34.5 \\
\hline Hodgkin's' disease & 92 & 7.6 \\
\hline Non-Hodgkin's lymphoma & 103 & 8.5 \\
\hline Burkitt's lymphoma & 202 & 16.6 \\
\hline Unspecified lymphoma & 23 & 1.9 \\
\hline CNS and intracranial and intraspinal neoplasms & 12 & 1.0 \\
\hline Ependymoma & 0 & 0.0 \\
\hline Astrocytoma & 2 & 0.2 \\
\hline Primitive neuroectodermal tumors & 8 & 0.7 \\
\hline Gliomas & 0 & 0.0 \\
\hline Unspecified CNS tumors & 2 & 0.2 \\
\hline Sympathetic nervous system & 21 & 1.7 \\
\hline Neuroblastoma & 19 & 1.6 \\
\hline Other & 2 & 0.2 \\
\hline Retinoblastoma & 16 & 1.3 \\
\hline Renal tumors & 46 & 3.8 \\
\hline Wilms' tumor & 41 & 3.4 \\
\hline Clear cell carcinoma & 2 & 0.2 \\
\hline Renal carcinoma & 2 & 0.2 \\
\hline Unspecified renal tumors & 1 & 0.1 \\
\hline Hepatic tumors & 21 & 1.7 \\
\hline Hepatoblastoma & 4 & 0.3 \\
\hline Hepatic carcinoma & 9 & 0.7 \\
\hline Unspecified malignant hepatic tumors & 8 & 0.7 \\
\hline Malignant bone tumors & 126 & 10.4 \\
\hline Osteosarcoma & 90 & 7.4 \\
\hline Chondrosarcoma & 21 & 1.7 \\
\hline Ewing's sarcoma & 12 & 1.0 \\
\hline Other specified malignant bone tumors & 2 & 0.2 \\
\hline Unspecified malignant bone tumors & 1 & 0.1 \\
\hline Soft-tissue sarcomas & 188 & 15.4 \\
\hline Rhabdomyosarcoma and embryonal sarcoma & 43 & 3.5 \\
\hline $\begin{array}{l}\text { Fibrosarcoma, neurofibrosarcoma, and other } \\
\text { fibromatous neoplasms }\end{array}$ & 23 & 1.9 \\
\hline Kaposi's sarcoma & 91 & 7.5 \\
\hline Other specified soft-tissue sarcomas & 18 & 1.5 \\
\hline Unspecified soft tissue sarcomas & 13 & 1.1 \\
\hline $\begin{array}{l}\text { Germ-cell, trophoblastic, and other gonadal } \\
\text { neoplasms }\end{array}$ & 117 & 9.6 \\
\hline Intracranial and intraspinal germ-cell tumors & 13 & 1.1 \\
\hline $\begin{array}{l}\text { Other and unspecified non-gonadal germ-cell } \\
\text { tumors }\end{array}$ & 7 & 0.6 \\
\hline
\end{tabular}

Table 1 continued

\begin{tabular}{lrr}
\hline & $\begin{array}{r}\text { Cases } \\
(\%)\end{array}$ \\
\hline Gonadal germ-cell tumors & $\begin{array}{l}\text { Total } \\
(1\end{array}$ & 5.8 \\
Gonadal carcinomas & 7 & 0.6 \\
Trophoblastic tumors & 11 & 0.9 \\
Other and unspecified malignant gonadal tumors & 8 & 0.7 \\
Carcinomas and other epithelial neoplasms & 167 & 13.7 \\
Adrenocortical carcinoma & 0 & 0.0 \\
Thyroid carcinoma & 17 & 1.4 \\
Nasopharyngeal carcinoma & 19 & 1.6 \\
Malignant melanoma & 7 & 0.6 \\
Skin carcinoma & 34 & 2.8 \\
Other and unspecified carcinoma & 90 & 7.4 \\
Other and unspecified malignant neoplasms & 53 & 4.4 \\
Other specified malignant tumors & 4 & 0.3 \\
Other unspecified malignant tumors & 49 & 3.8 \\
\hline
\end{tabular}

When comparing our Kijabe database with several of the other previous African databases-including the Makata database from Kenya [8], Patil's database from Zambia [10], and Mukiibi's database from Malawi [7] there are more similarities. The lymphomas are much more prominent, especially Burkitt's and non-Hodgkin's lymphoma which take up the top two positions on all the African databases. In the Malawi database, Burkitt's lymphoma is included in the non-Hodgkin's lymphomas (54.9\%). Kaposi's sarcoma is prominent in all the African databases, being the fourth most common diagnosis in three of them. Epithelial carcinoma is represented on three of the four "top ten" lists. It is more highly represented as the fourth most common diagnosis in Malawi (5.7\%), when compared to the two Kenyan databases in which it is number $9(2.8 \%$, Kijabe) and 8 (2.6\%, Makata), respectively.

There are some differences, however, between our spectrum and that of the other African databases. Hodgkin's lymphoma appears in all five databases with slightly higher occurrence in our database (7.6\%) as compared with the others, which have roughly equivalent frequency at around $4 \%$ each. Osteosarcoma was number 5 in frequency in our database (7.4\%) as compared with the other four databases in which it came in 9 or 10 with equal frequency, around $2 \%$ of malignancies represented. Gonadal germ-cell tumors (5.6\%) and fibrosarcoma (1.9\%) only made the "top ten list" in our database alone. Also, the retinoblastomas, which appear prominently as the second or third most common diagnosis in the other African databases (around $11 \%$ of the total in each), ranks number 13 in frequency in our database (1.3\%).

When examining gender distribution as seen in Table 3, Hodgkin's and non-Hodgkin's lymphomas, chondrosarcoma, 


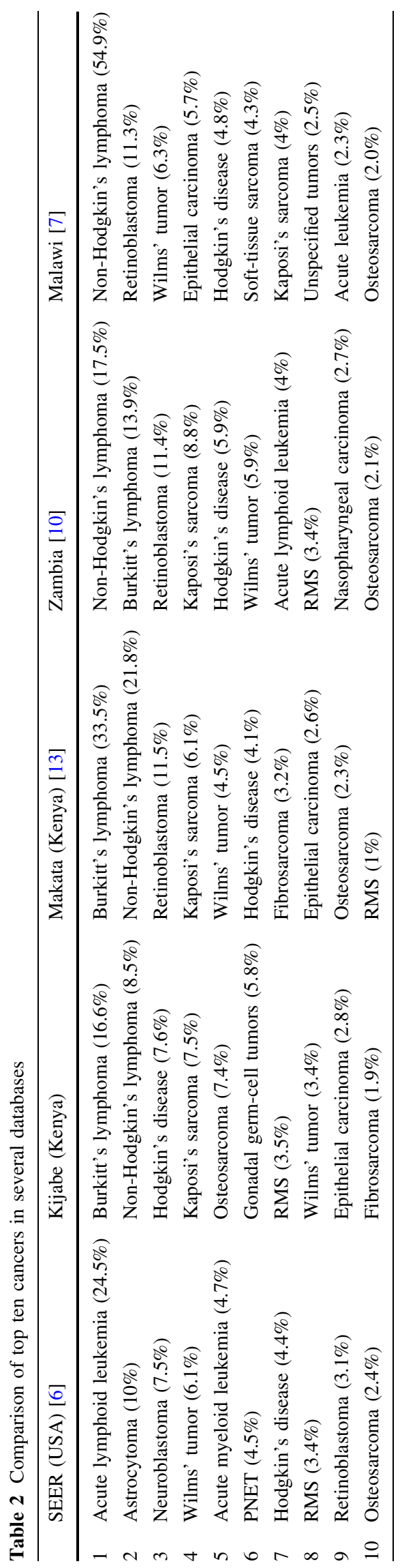

and Kaposi's sarcoma had a strong male predominancewhile the gonadal germ-cell tumors had a strong female predominance. There was a slight male predominance in Burkitt's lymphoma and a slight female predominance in rhabdomyosarcoma and nephroblastoma.

As expected, nephroblastoma, neuroblastoma, and retinoblastoma presented at younger ages $(<5$ years), while Hodgkin's, osteosarcoma, gonadal germ-cell tumors, epithelial carcinoma, and fibrosarcoma presented in older children ( $>10$ years).

\section{Discussion}

This analysis is clearly not meant to document the true disease incidence in the Kenyan population-but rather, a cross-sectional analysis of the observed pediatric cancers presenting to mission and other regional hospitals in Kenya. Any inferences to population-based data must be very cautious. Also, no meaningful inferences can be made regarding specific ethnic groups because these are widely mixed in the Kenyan population and most referring hospitals serve multiple groups. Moreover, Kijabe Hospital is not specifically a referral center for the treatment of pediatric cancers, and many of the cases included were referred to cancer centers for further management. Finally, it was not possible to establish a geographic distribution of disease from our database. Although the institution of origin for each specimen was known, each of these institutions draws patients from varied areas of the country, and the regional distribution where each patient lived was not available.

The spectrum of pediatric cancers in our database clearly differs from that observed in western pediatric oncologic databases [5, 6]. Although the database had many similarities with previous African databases, there are some key differences which may help provide greater understanding of the true distribution of disease. Discussion will follow the 12 histopathologic categories specified by the ICCC [4].

First, leukemias were underrepresented in our database as compared with the western pediatric databases. This decreased frequency has been previously described in the African population [2], although it is unclear how much of this difference is related to a truly lower disease incidence as opposed to underdiagnosis. Acute leukemias may also be underrepresented in our sample simply because not all children who are diagnosed with leukemia and initiated on therapy in our setting undergo a bone marrow biopsy. Some cases are diagnosed and treated based on clinical suspicion and peripheral blood evaluation, which would not be reflected in our database. 
Table 3 Characteristics of top 12 pediatric cancers

\begin{tabular}{lcll}
\hline & $\begin{array}{l}\text { Mean } \\
\text { age }\end{array}$ & $\begin{array}{l}\text { Percent } \\
\text { male }\end{array}$ & $\begin{array}{l}\text { Most common } \\
\text { location }\end{array}$ \\
\hline Burkitt's lymphoma & 8 & 53.5 & Abdomen \\
Non-Hodgkin's lymphoma & 9 & 69.9 & Abdomen \\
Hodgkin's lymphoma & 11 & 76.1 & Neck node \\
Kaposi's sarcoma & 9 & 64.1 & Neck/neck node \\
Osteosarcoma & 13 & 49.4 & Femur \\
Gonadal germ-cell tumors & 12 & 18.3 & Ovary \\
Rhabdomyosarcoma & 9 & 44.2 & Leg \\
Wilms' tumor & 4 & 46.3 & Kidney \\
Skin carcinoma & 10 & 50 & Head/neck \\
Fibrosarcoma & 11 & 47.8 & Leg \\
Chondrosarcoma & 14 & 63.6 & Leg \\
Neuroblastoma & 5 & 52.6 & Retroperitoneum \\
\hline
\end{tabular}

The lymphomas are strongly represented in all the African databases [7, 10, 13] and took up the three most frequently occurring diagnoses in our database. Burkitt's lymphoma is the most common pediatric cancer in subSaharan Africa, with a specific clustering in Western Kenya $[13,16]$. Kenya lies within the "lymphoma belt" which stretches across the African continent on both sides of the equator. This cancer has well-established links to the Epstein Barr Virus, holoendemic malaria [16], and HIV infection [19, 20], all of which are highly prevalent in Kenya. Non-Hodgkin's disease has also been linked with HIV infection [19], while Hodgkin's disease has been linked strongly to EBV infection [18]. A male predominance for the lymphomas has likewise been described [2]. The high frequency of lymphoma in the Kenyan population provides the unique opportunity to gain a deeper understanding of this disease group.

Due to cost and lack of availability of three-dimensional imaging techniques, as well as qualified surgeons for biopsy, central nervous system tumors are likely underdiagnosed and therefore underrepresented in our database as compared with western statistics [5, 6]. Many of these cases would be referred to larger centers for diagnosis and treatment. However, a truly lower incidence in Africa for brain tumors cannot be ruled out.

Sympathetic nervous system tumors, such as neuroblastoma, had a lower incidence in our database as compared with western statistics. This was consistent with the other African databases [7, 10, 13]. The lowest rates in the world for sympathetic tumors have previously been shown to be in sub-Saharan Africa [2]-for reasons that remain unknown.

Retinoblastoma had a significantly lower occurrence in our database compared with the other African databases, including the Makata database from Kenya. This may be a result of sample bias, due to the fact that Kijabe Hospital and its affiliates within the pathology network do not have ophthalmology departments. The true incidence of this diagnosis is likely therefore underrepresented.

Renal tumors such as Wilms' were relatively equally represented in the various databases, and no particular trends were elucidated.

Liver tumors are rare in the pediatric population, and subsequently had very little representation on our database. This may be partially due to underdiagnosis in the Kenyan context, due to lack of access to three-dimensional imaging and biopsy. Moreover, some of the harder-to-diagnose hepatoblastomas were likely diagnosed as "unspecified", thus accounting for hepatocellular carcinomas being more prevalent than hepatoblastomas. Patient charts were not available for review in this study, and therefore no correlation can be made to hepatitis or HIV status in these patients.

Bone tumors were strongly represented in our database as compared with the other studies. This increased frequency is partially sample bias, due to a high number of samples coming from the neighboring pediatric orthopedic hospital. Osteosarcoma, for example, had roughly equivalent frequency in all databases but our own. However, previous reports of worldwide incidence of bone tumors at $5 \%$ of pediatric cancers are more consistent with our database [2].

Soft tissue sarcomas were strongly represented with rhabdomyosarcoma, fibrosarcoma, and Kaposi's sarcoma all among the top ten most frequent diagnoses. The incidence of Kaposi's sarcoma, which was strongly represented on all four African databases, has increased in recent years due to the AIDS epidemic in Africa $[2,11,20]$.

Germ-cell cancers, which typically represent less than $3 \%$ of childhood cancers [2], were strongly represented among our data - especially dysgerminomas and teratomas of the ovary. The reasons for the high incidence in our population are unclear.

Epithelial cancers are a varied group in the pediatric population, most commonly dominated by thyroid carcinoma, malignant melanoma, and nasopharyngeal carcinoma [6]. Typically, adult epithelial cancers such as breast, intestinal, and prostate have little representation in the pediatric population and are therefore grouped together as "other" or "unspecified" in the pediatric classification scheme [2, 4]. In our database, however, we had a strong representation of these diagnoses, especially in older children. Although low in numbers, breast (8), esophageal (8), gastric (7), intestinal (1), and prostatic (2) cancers were all present. By far the largest contributor to the 90 patients in the category for "other and unspecified carcinoma" was undifferentiated carcinomas and adenocarcinomas. There 
was a trend towards higher incidence of skin carcinomas, especially in older children. This trend was also seen in several of the other African databases [7, 13] and warrants further investigation.

There were some inherent flaws in the database which require specific mention. The database is a collection of pathologic diagnoses, isolated in the majority of cases from patient charts and therefore not offering the availability for chart review to confirm demographic data. Therefore, any errors in data entry could affect the final results. The main result of such errors is likely not incorrect inclusion of a patient, but rather omission of patients because of inability to identify their age.

The spectrum of pediatric cancer in Kenya is unique in the world. Even within the country, cancer incidence varies among tribes and geographic areas $[13,14,16]$. This presents an opportunity for further research and therapeutic advancements for the children in Kenya, many of whom currently have limited access to care. A collaborative effort among mission hospitals, regional hospitals, and major teaching centers through a national pediatric cancer database would greatly benefit such efforts in the future.

\section{References}

1. Hutt MSR, Burkitt D (1965) Geographical distribution of cancer in East Africa: a new clinicopathological approach. Br Med J 2:719-722

2. Stiller CA, Parkin DM (1996) Geographic and ethnic variations in the incidence of childhood cancer. Br Med Bull 52(2):682-703

3. Birch JM, Marsden HB (1987) A classification scheme for childhood cancer. Int J Cancer 40:620-624

4. Kramarova E, Stiller CA, Ferlay J et al (eds) (1996) The international classification of childhood cancer (1996 revision). IARC Technical Report, No. 29. IARC, Lyon

5. Grovas A, Fremegen A, Rauck A, Ruymann F, Hutchinson CL, Winchester DP, Menck HR (1997) The National Cancer Data Base Report on patterns of childhood cancers in the United States. Cancer 80(12):2321-2332
6. National Cancer Institute (1975-1995) Cancer incidence and survival among children and adolescents: United States SEER Program

7. Mukiibi JM, Banda L, Liomba NG, Sungani FC, Parkin DM (1995) Spectrum of childhood cancers in Malawi 1985-1993. East Afr Med J 72(1):25-29

8. Carneiro PM, Kalokola FM, Kaaya EE (1998) Paediatric malignancies in Tanzania. East Afr Med J 75(9):533-535

9. Ocheni S, Okafor CO, Emodi IJ, Ibegbulam OG, Olusina DB, Ikefuna AN, Nnakenyi EF (2005) Spectrum of childhood malignancies in Enugu, Nigeria (1999-2004). Afr J Med Med Sci 34(4):371-375

10. Patil PS, Elem B, Gwavava NJ, Urban MI (1992) The pattern of paediatric malignancy in Zambia (1980-1989): a hospital-based histopathological study. J Trop Med Hyg 95(6):434

11. Chintu C, Athale UH, Patil PS (1995) Childhood cancers in Zambia before and after the HIV epidemic. Arch Dis Child 73:100-105

12. Mwanda OW (1999) Cancers in children younger than age 16 years in Kenya. East Afr Med J 76(1):3-9

13. Makata AM, Toriyama K, Kamidigo NO, Eto H, Itakura H (1996) The pattern of pediatric solid malignant tumors in western Kenya, East Africa, 1979-1994: An analysis based on histopathologic study. Am J Trop Med Hyg 54(4):343-347

14. Mwanda OW, Rochford R, Moormann AM, MacNeil A, Whalen C, Wilson ML (2004) Burkitt's lymphoma in Kenya: geographical, age, gender, and ethnic distribution. East Afr Med J 8(Suppl):S68-S77

15. Mwanda WO, Orem J, Remick SC, Rochford R, Whalen C, Wilson ML (2005) Clinical characteristics of Burkitt's lymphoma from three regions in Kenya. East Afr Med J 82(9 Suppl):S135-S143

16. Rainey JJ, Omena D, Sumba P, Moormann AM, Rochford R, Wilson ML (2006) Spatial clustering of endemic Burkitt's lymphoma in high-risk regions of Kenya. Int J Cancer 120:121-127

17. Kusuda M, Toriyama K, Kamidigo N, Itakura H (1998) A comparison of epidemiologic, histologic, and virologic studies on Hodgkin's disease in Western Kenya and Nagasaki, Japan. Am J Trop Med Hyg 59(5):801-807

18. Weinreb M, Day PJR, Niggli F, Green EK, Nyong'o AO, Othieno-Abinya NA, Riyat MS, Raafat R, Mann JR (1996) The consistent association between Epstein-Barr virus and Hodgkin's disease in children in Kenya. Blood 87:3828-3836

19. Biggar RJ, Frisch M, Goedert JJ et al (2000) Risk of cancer in children with AIDS. AIDS-Cancer Match Registry Study Group. JAMA 284(2):205-209

20. Parkin DM, Wabinga $H$, Nambooze $S$, Wabwire-Mangen $F$ (1999) AIDS-related cancers in Africa: maturation of the epidemic in Uganda. AIDS 13(18):2589-2590 